\title{
Structural and Optical Properties of Nanocrystalline 3,4,9,10-Perylene-Tetracarboxylic-Diimide Thin Film
}

\author{
M. M. El-Nahhas, ${ }^{1}$ H. Abdel-Khalek, ${ }^{2}$ and E. Salem ${ }^{2}$ \\ ${ }^{1}$ Physics Department, Faculty of Education, Ain Shams University, Roxy, Cairo 11757, Egypt \\ ${ }^{2}$ Physics Department, Faculty of Science, Suez Canal University, Ismailia 41522, Egypt
}

Correspondence should be addressed to E. Salem, eman_m_salem@yahoo.com

Received 8 March 2012; Accepted 24 April 2012

Academic Editor: Victor V. Moshchalkov

Copyright (C) 2012 M. M. El-Nahhas et al. This is an open access article distributed under the Creative Commons Attribution License, which permits unrestricted use, distribution, and reproduction in any medium, provided the original work is properly cited.

\begin{abstract}
Thin films of nanocrystalline 3,4,9,10-perylene-tetracarboxylic-diimide (PTCDI) were prepared on quartz substrates by thermal evaporation technique. The structural properties were identified by transmission electron microscopy (TEM) and the Xray diffraction (XRD). The optical properties for the films were investigated using spectrophotometric measurements of the transmittance and reflectance at normal incidence of light in the wavelength range from 200 to $2500 \mathrm{~nm}$. The optical constants (refractive index $n$ and absorption index $k$ ) were calculated and found to be independent on the film thickness in the measured film thickness range $117-163 \mathrm{~nm}$. The dispersion energy $\left(E_{d}\right)$, the oscillator energy $\left(E_{o}\right)$, and the high-frequency dielectric constant $\varepsilon_{\infty}$ were obtained. The energy band model was applied, and the types of the optical transitions responsible for optical absorption were found to be indirect allowed transition. The onset and optical energy gaps were calculated, and the obtained results were also discussed.
\end{abstract}

\section{Introduction}

Since the discovery of perylene dyes like 3,4,9,10-perylenetetracarboxylic-diimide (PTCDI, Figure 1) in 1913, they are mainly used as technical pigments. More recently, perylene molecules and their derivatives have attracted more and more attention in the past decade due to not only their outstanding thermal and photochemical stabilities $[1,2]$, but also their large application potential in organic optoelectronic or electronic devices [3-11], such as field effect transistors, solar cells [12-20], light-harvesting arrays [2125], and light-emitting diodes [26-30]. The layered growth of these planar molecules makes it possible to prepare fairly well ordered thin films of perylenes on different substrates [31]. The charge transport and luminescence properties strongly depend on the structural properties of these thin films $[32,33]$.

For organic materials relevant for light-emitting diodes, a better control over device performance may be achieved by changing the morphology of the organic film, for example, by going down to nanoscale crystallites size.
In this paper, the structural properties of PTCDI films grown at room temperature on quartz substrate were assessed by means of X-ray and transmission electron microscopy (TEM). We have investigated the optical properties of the 3,4,9,10-perylene-tetracarboxylic-diimide nanocrystalline thin films. The optical constants such as refractive index, extinction coefficient, dielectric constant, optical band, and dispersion energy are determined.

\section{Experimental Details}

The PTCDI films have been prepared by conventional thermal evaporation in vacuum chamber at pressure of about $p=2 \times 10^{-4} \mathrm{~Pa}$. PTCDI powder (99.99\%, Sigma Aldrich Co.) was loaded into a molybdenum cell with nozzle of $5 \mathrm{~mm}$ in diameter on the top. The flat quartz substrates were located above $15 \mathrm{~cm}$ from the source. The substrates were carefully cleaned by putting them in chromic acid for $15 \mathrm{~min}$, and then they were washed several times with distilled water. After that, the substrates were rinsed by isopropyl alcohol. The substrates were dried in a steam of dry nitrogen, and finally, 


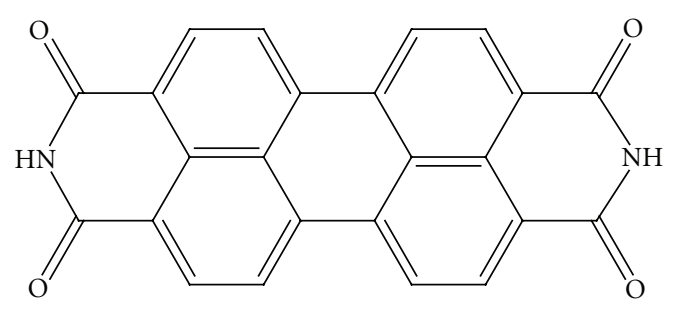

Figure 1: 3,4,9,10-perylene-tetracarboxylicacid-diimide, PTCDI.

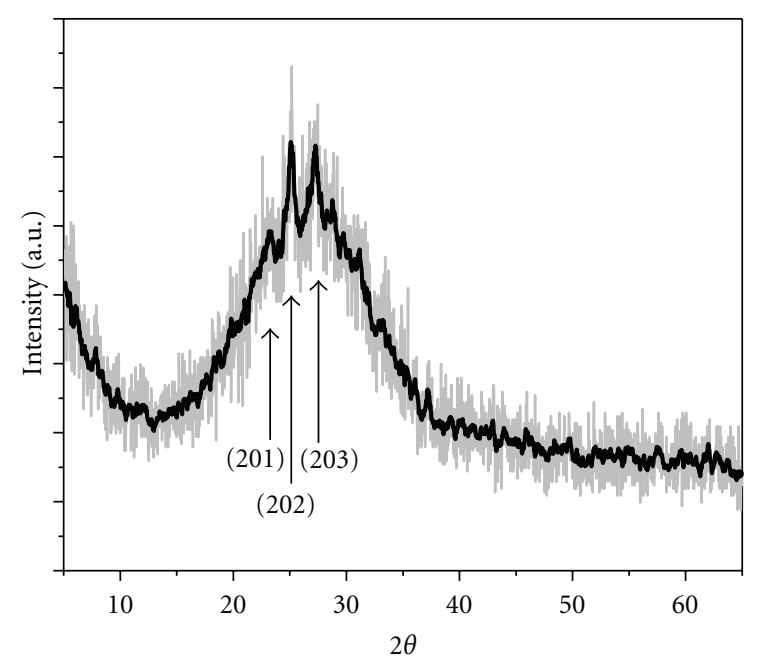

FIGURE 2: XRD spectra of 3,4,9,10-perylene-tetracarboxylicaciddiimide, PTCDI, as-deposited thin.

they were cleaned by atomic bombardment in an initial stage of evacuation. The film thickness was controlled by using a quartz crystal thickness monitor and subsequently calibrated by Tolansky's method [34]. All films were deposited at room temperature, and the rate of deposition was adjusted at $2.5 \mathrm{~nm} / \mathrm{s}$ by the above-mentioned thickness monitor.

Room temperature XRD measurements (X'Pert Philips $\mathrm{X}$-ray diffractometer) were carried out using $\mathrm{CuK}_{\alpha 1}$ radiation in the $(\theta-2 \theta)$ geometry. The spectra of the films were scanned over the range of $5^{\circ}$ to $60^{\circ}(2 \theta)$, with a step rate of $0.02^{\circ}(2 \theta)$ and a fixed counting time of $10 \mathrm{~s}$ for each step, in order to obtain spectra with sufficient signal-to-noise ratio. TEM mages were captured using Joel JEM-1400 transmission electron microscope with high voltage range from $40 \mathrm{kV}$ up to $120 \mathrm{kV}$.

The transmittance $T(\lambda)$ and reflectance $R(\lambda)$ spectra of PTCDI films were measured at normal incidence at room temperature in the spectral range of $200-2500 \mathrm{~nm}$ by using a computer-aided double-beam spectrophotometer (JASCO model V-570 UV-VIS-NIR). A blank quartz substrate identical to that used for the sample was used as a reference for the transmittance scan. However, the reflectance scan was taken at an incident angle of $5^{\circ}$ with $\mathrm{Al}$ mirror as a reference.

In order to calculate the refractive index $(n)$ and the absorption index $(k)$ of the thin films at different wave-
TABLE 1: X-ray for PTCDI films.

\begin{tabular}{lcc}
\hline $2 \theta$ & $d$ & $\mathrm{hkl}$ \\
\hline 24.88 & 3.57 & $(201)$ \\
26.97 & 3.30 & $(202)$ \\
30.29 & 2.94 & $(203)$ \\
\hline
\end{tabular}

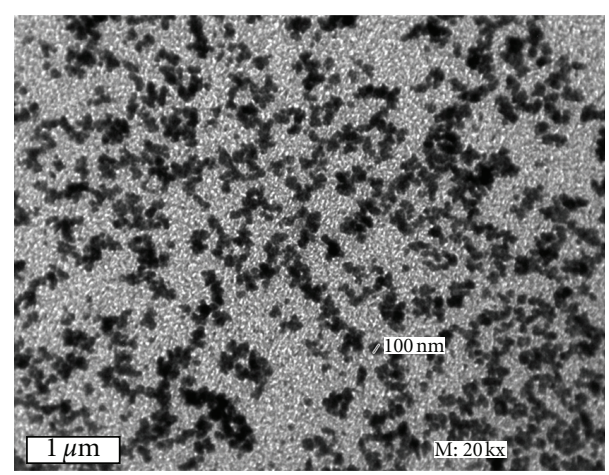

FIGURE 3: TEM image of 4,9,10-perylene-tetracarboxylicaciddiimide, PTCDI, film.

lengths, the following equations are applied:

$$
\begin{gathered}
n=\frac{1+R}{1-R}+\sqrt{\frac{4 R}{(1-R)^{2}}-k^{2}}, \\
\alpha=\frac{1}{d} \ln \left[\frac{(1-R)^{2}}{2 T}+\sqrt{\frac{(1-R)^{4}}{4 T^{2}}+R^{2}}\right], \\
k=\frac{\alpha \lambda}{4 \pi},
\end{gathered}
$$

where $\alpha$ is the absorption coefficient. When the thickness of film $(d)$ is known, then the computation can be carried out and the optical constants, $n$ and $k$, can be calculated. The experimental error was taken as $\pm 2 \%$ for film thickness, $\pm 1 \%$ for $T$ and $R, 4 \%$ for $n$, and $3 \%$ for $k$.

\section{Results and Discussion}

3.1. Structure Investigation. X-ray diffraction (XRD) of PTCDI films was taken in a $(2 \theta)$ range from $5^{\circ}$ to $70^{\circ}$, and its spectrum is presented in Figure 2. As shown in this figure, the pattern has many diffraction peaks with different intensities indicating that the film is polycrystalline/nanocrystalline structure. The analysis of XRD diffraction data at room temperature reveals that the film possesses an orthorhombic cell symmetry (PNA21 space group), with the following lattice parameters: $a=7.368 \AA, b=10.924 \AA, c=14.777 \AA$, and $\alpha=\beta=\gamma=90^{\circ}$. More details are illustrated in Table 1 .

The TEM image of PTCDI film is illustrated in Figure 3. The image shows that the film exhibits a rough surface with random distribution of nanocluster over a top surface. The clusters have an average diameter of about $100 \mathrm{~nm}$.

3.2. Optical Characterizations. The spectral distributions of $T$ and $R$ for the PTDCI film measured at normal incidence 


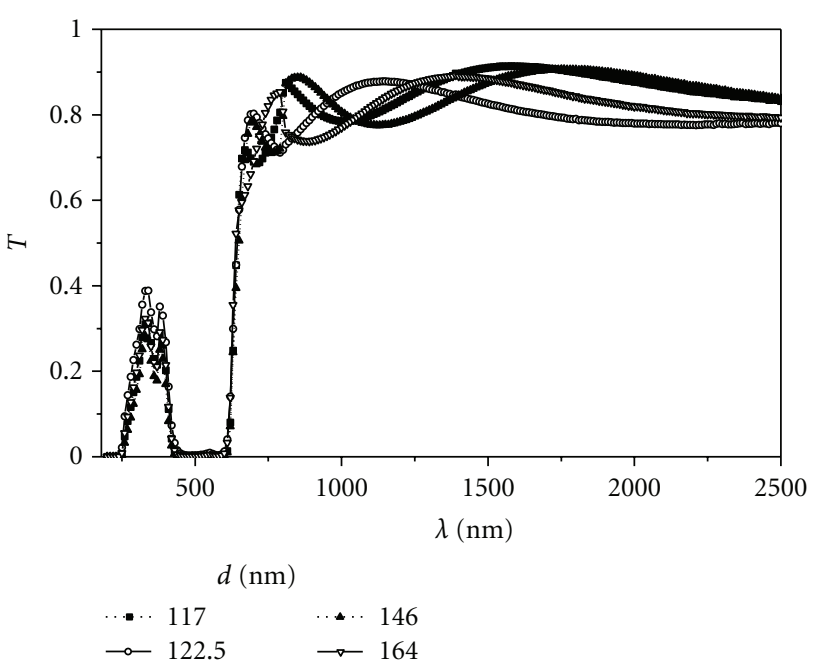

(a)

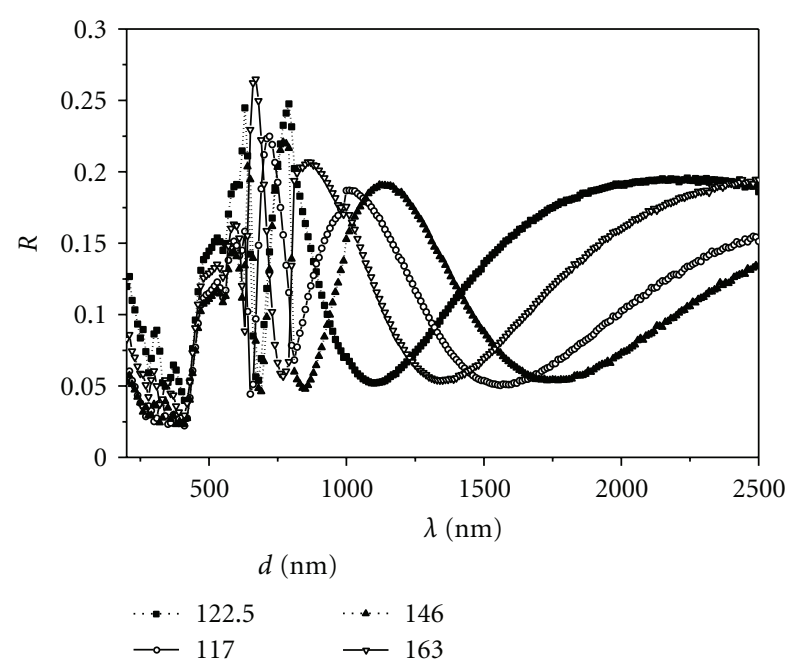

(b)

FIGURE 4: The spectra of the prepared PTCDI films (a) transmission and (b) reflection.

are shown in Figure 4. It is quite clear from the figure that at wavelength $\lambda>800 \mathrm{~nm}$, that is, the films are transparent, and no light is scattered or absorbed, (nonabsorbing region) $T+$ $R=1$. The inequality $R+T<1$ at shorter wavelengths $\lambda<$ $800 \mathrm{~nm}$ implies the existence of absorption, that is, absorbing region.

The calculated optical constants were found to be independent of the film thickness range of $117-163 \mathrm{~nm}$ within the estimated experimental errors. The variation in the dispersion curve of refractive index $n$ for the PTCDI films, plotted from the mean values of various film thicknesses in the spectral range of $200-2500 \mathrm{~nm}$, is presented in Figure 5. It is observed that there is an anomalous dispersion at $\lambda<$ $900 \mathrm{~nm}$ in exhibiting various peaks. The anomalous dispersion behavior obeys the multioscillator Lorentz-Lorenz model [35]. The normal dispersion is observed at $\lambda>$ $900 \mathrm{~nm}$.

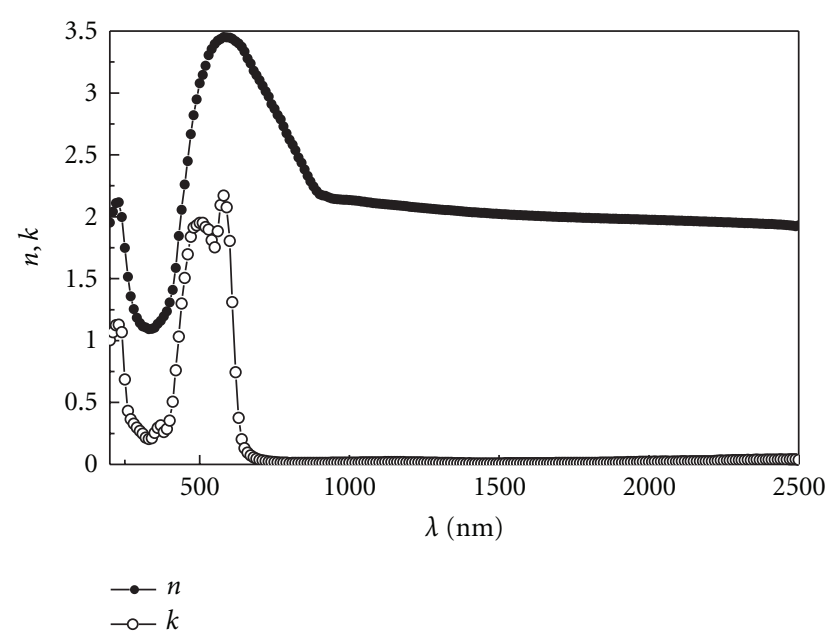

FIGURE 5: Refractive index and extinction coefficient of the PTDCI film.

TABLE 2: Dispersion parameters of the PTDCI films.

\begin{tabular}{cccccc}
\hline PTDCI & $\varepsilon_{\infty}$ & $\varepsilon_{L}$ & $\begin{array}{c}N / M^{*} \\
\left(10^{45} \mathrm{~kg}^{-1} \mathrm{~m}^{-3}\right)\end{array}$ & $\begin{array}{c}E_{0} \\
(\mathrm{eV})\end{array}$ & $\begin{array}{c}E_{d} \\
(\mathrm{eV})\end{array}$ \\
\hline 2.73 & 4.22 & 8.61 & 2.41 & 6.59 \\
\hline
\end{tabular}

The normal dispersion of refractive index has been analyzed by applying the single-oscillator model; the wellknown Wemple and DiDomenico is given by equation [36, 37]

$$
n^{2}-1=\frac{E_{d} E_{o}}{\left(E_{o}^{2}-(h \nu)^{2}\right)}
$$

where $E_{o}$ is the single-oscillator energy, and $E_{d}$ is the dispersion energy. By plotting $\left(n^{2}-1\right)^{-1}$ versus $(h \nu)^{2}$, Figure 6, and fitting the data to a straight line, $E_{o}$ and $E_{d}$ can be determined from the intercept, $E_{o} / E_{d}$, and the slope, $-1 / E_{o} E_{d}$. The oscillator energy $E_{o}$ can be considered as an average energy gap and was found to be in proportion to the optical energy gap $E_{g}$, which agrees well with the relation $E_{o} \approx 2.3 E_{g}$ reported in [38].

The calculated values of $E_{o}$ and $E_{d}$ as well as the corresponding high-frequency dielectric constant $\left(\varepsilon_{\infty}=n_{\infty}^{2}\right)$ for PCDTI films are summarized in Table 2.

The relation between the real part of the dielectric constant $\varepsilon_{1}$ and wavelength $(\lambda)$ is given by $[39,40]$

$$
\varepsilon_{1}=n^{2}=\varepsilon_{L}-\frac{e^{2}}{\pi c^{2}} \frac{N}{m^{*}} \lambda^{2},
$$

where $\varepsilon_{L}$ is the lattice dielectric constant, and $N / m^{*}$ is the ratio of free carrier concentration to the effective mass. The relation between $n^{2}$ and $\lambda^{2}$ is shown in Figure 7 which verifies the linearity of the above equation. The values of $\varepsilon_{L}$ and $\left(\mathrm{N} / \mathrm{m}^{*}\right)$ are determined from the extrapolation of this plot to $\lambda^{2}=0$ and from the slope of the graph, respectively, for the PTDCI film (see Table 2). As observed from Table 2, the lattice dielectric constant $\varepsilon_{L}$ is higher than the value of 


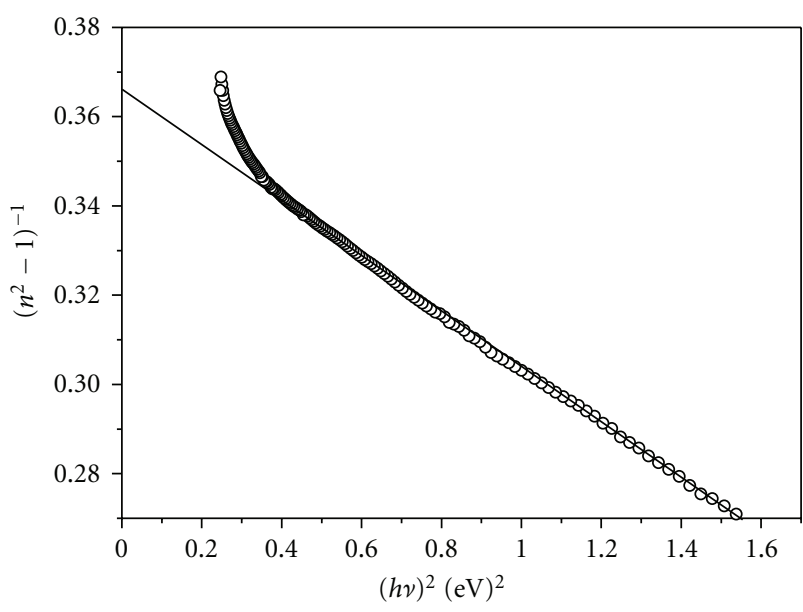

FIgUre 6: The plot of $\left(n^{2}-1\right)^{-1}$ against $(h \nu)^{2}$ for PTDCI films.

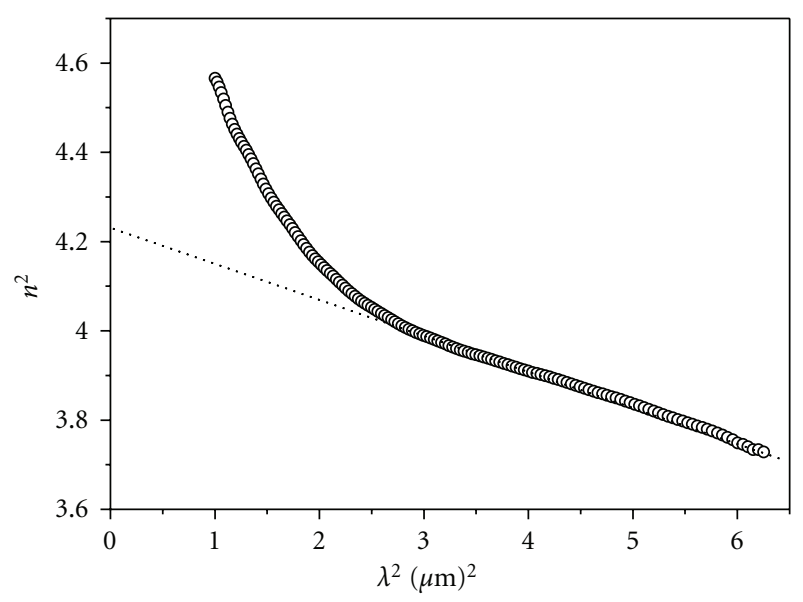

Figure 7: $n^{2}$ versus $\lambda^{2}$ for the PTDCI films.

the high-frequency dielectric constant $\varepsilon_{\infty}$. This change may be attributed to the free carrier contribution to the dispersion.

3.3. Optical Energy Gap. The spectral distribution of the absorption coefficient $(\alpha)$ for PTCDI films is shown in Figure 8. A close examination of the absorption band in the visible region, known as $Q$-band, appears in the region between 2 and $3 \mathrm{eV}$. The $Q$-band consists of one shoulder at $2.5 \mathrm{eV}$ which has been assigned to $\pi-\pi^{*}$ transitions, and one peak at $2.14 \mathrm{eV}$ has been explained as exciton peak. It can also be noticed that the splitting characteristic (Davydov splitting), $\Delta Q$, equals $0.36 \mathrm{eV}$. In the UV spectral region at $3.35 \mathrm{eV}$, there is a weak band called $B$-band. This is due to the electronic transition from $\pi-\pi^{*}$. The weak absorption plateau at $4 \sim 4.75 \mathrm{eV}$ is called $N$-band, which has been attributed to the charge transfer (CT) from the $s p_{z}$ mixing orbital to the electron system of the macrocyclic ring of the PTCDI. The $C$-band is another region of absorption at $5.6 \mathrm{eV}$ due to $\pi-\pi^{*}$ transition.

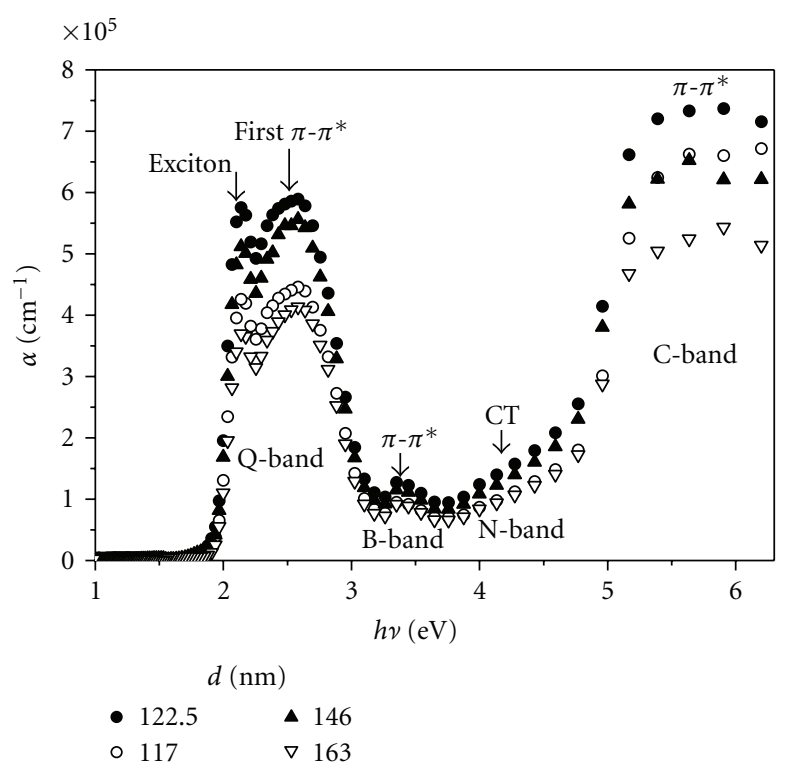

FIgURE 8: Optical absorption spectra of PTCDI thin films.

The absorption coefficient $\alpha$ is well described by the relation $[40]$

$$
(\alpha h v)^{r}=G\left(h v-E_{g} \pm E_{\mathrm{ph}}\right)
$$

where $E_{g}$ is the value of the optical bandgap corresponding to transitions indicated, $E_{\mathrm{ph}}$ is the energy of phonons assisted indirect transitions, and the factor $(G)$ depends on the transition probability which can be assumed to be constant within the optical frequency range.

To obtain the film optical energy gap and determine the nature of the optical transitions involved, the dependence of the absorption coefficient on the photon energy should be analyzed near fundamental absorption edges within the framework of one electron theory [40]. The dependence of $(\alpha h \nu)^{r}$ on photon energy $(h \nu)$ was plotted for different values of $r$, where $r$ is a constant which determines type of the optical transition $(r=2)$ for allowed direct transitions and $(r=1 / 2)$ for allowed indirect transitions, the best fit was obtained for $r=1 / 2$, indicate indirect transition, Figure 9. The extrapolation of the straight line graphs to zero absorption will give the values of the onset and energy gap. It is worth to mention the transport gap "HOMO-LOMO gap," $E_{t}$. This trap energy is the minimum energy formation of a separated, uncorrelated free electron and hole and associated with the transport of single particles in the solid. The optical gap $E_{g}$, on the other hand, corresponds to the onset of optical absorption and formation of a bound electron-hole pair, or exciton. $E_{t}$ is larger than $E_{g}$ by a significant amount corresponding to the binding energy of the exciton, $E_{B}$, the consensus from experimental results was obtained over the past few years with various techniques, as well as from theory, that is, $E_{B}=0.5-1.5 \mathrm{eV}$ in molecular solid [41]. The magnitudes of the obtained values of $E_{g}, E_{t}$, and $E_{\mathrm{ph}}$ are listed in Table 3. 


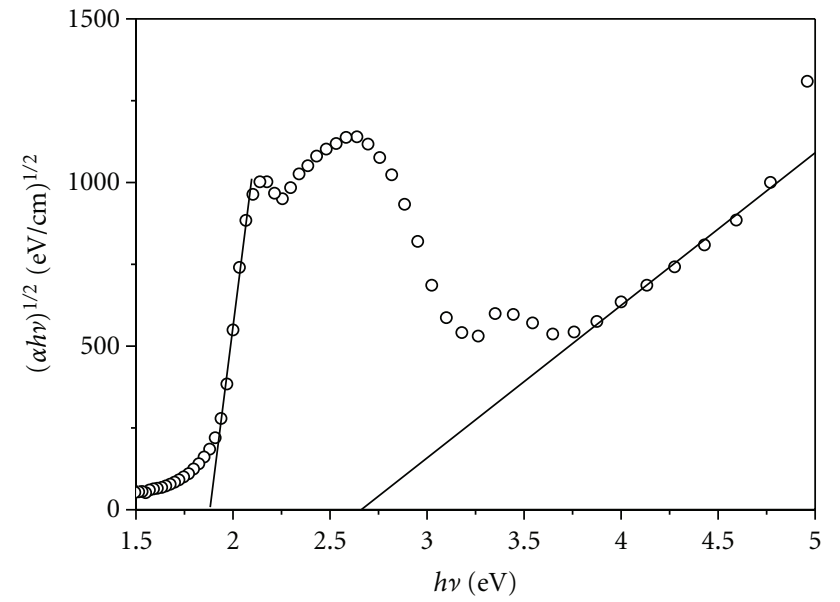

FIGURE 9: The plot of $(\alpha h \nu)^{1 / 2}$ versus the incident photoenergy $(h \nu)$ for PTCDI films.

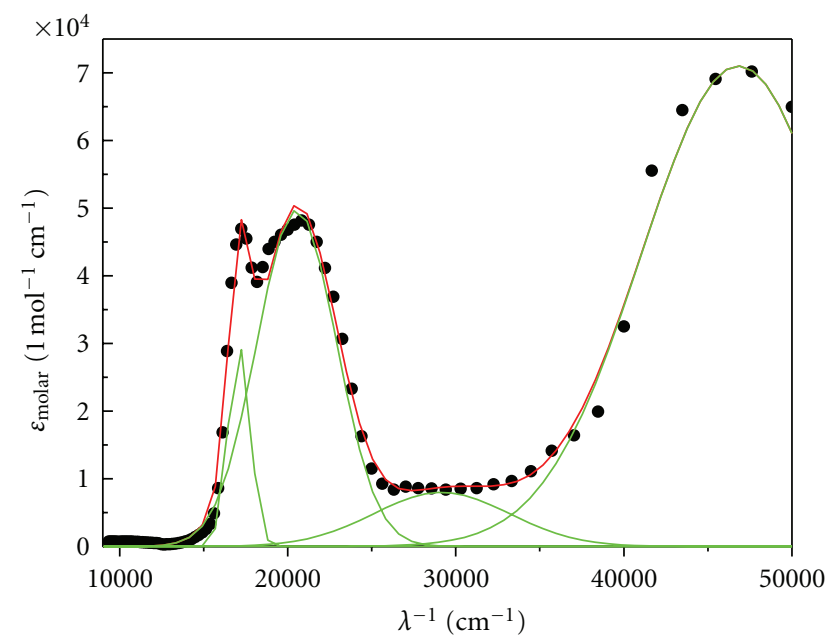

FIGURE 10: Molar extinction coefficient versus wave number (circle point) for as-deposited thin films of (PTCDI) fitted using the Gaussian model (solid line) assuming two oscillator components. The dashed lines present the decomposition of the molar extinction spectrum on particular oscillatory transitions.

TABLE 3: Values of energy gaps of PTCDI thin films according to the band transition.

\begin{tabular}{|c|c|c|c|}
\hline \multirow{2}{*}{ PTCDI } & \multicolumn{2}{|c|}{ Transport gap "HOMO-LOMO gap" } & \multirow{2}{*}{$\begin{array}{c}\text { Onset gap } \\
E_{\mathrm{ph}}(\mathrm{eV})\end{array}$} \\
\hline & $E_{t}^{\text {ind }}(\mathrm{eV})$ & $E_{g}^{\text {ind }}(\mathrm{eV})$ & \\
\hline As deposited & $2.68 \pm 0.01$ & $1.89 \pm 0.02$ & 0.03 \\
\hline
\end{tabular}

It is useful to relate the absorption coefficient $(\alpha)$ to the molar extinction $\left(\varepsilon_{\text {molar }}\right)$, which is often used to describe the absorption of light by nonsolid molecular media, and in the solid, the absorption coefficient can be written in the form $[42,43]$

$$
\alpha=2303\left(\frac{\rho}{m}\right) \varepsilon_{\text {molar }}
$$

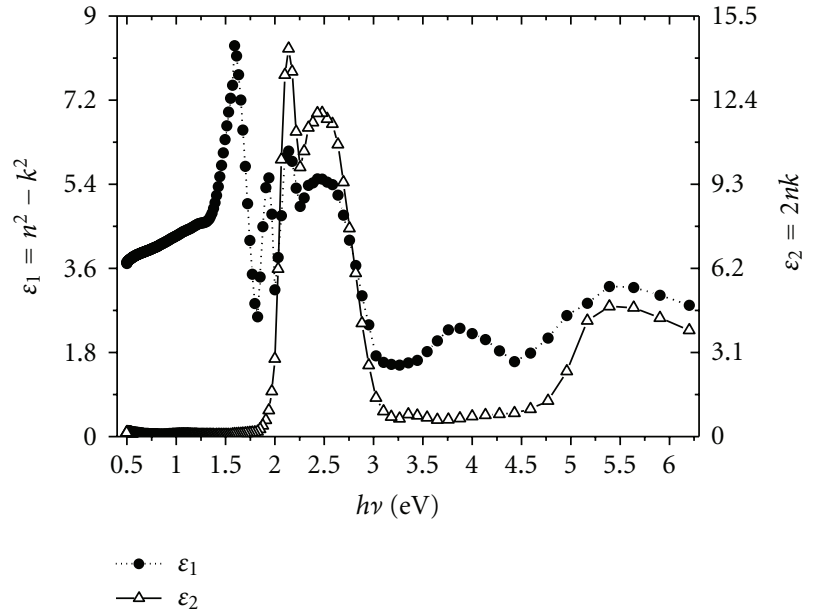

FIGURE 11: Plot of $\varepsilon_{1}$, the real dielectric constant, and $\varepsilon_{2}$, the imaginary dielectric constant, versus photon energy for PTDCI films, as indicated in the figure.

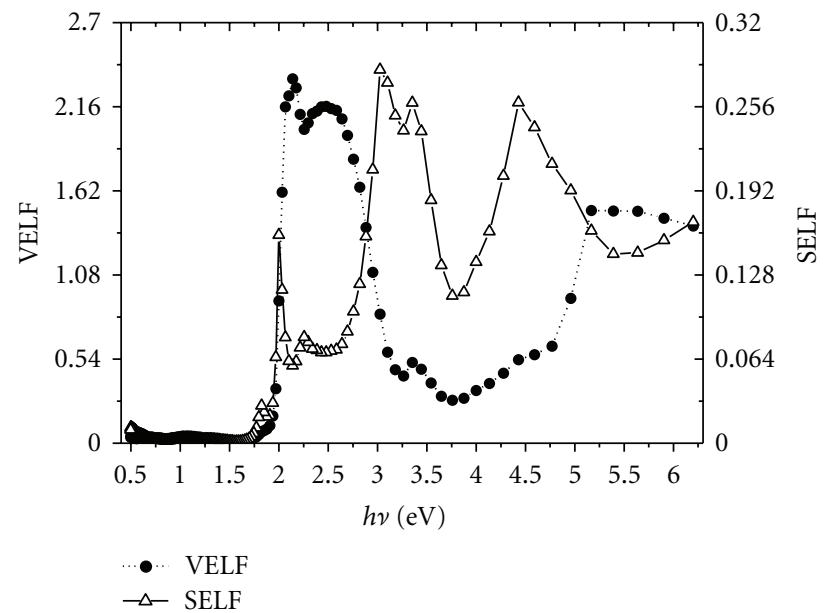

FIGURE 12: The volume energy loss functions and the surface energy loss functions against $h v$ for PTDCI films.

Figure 10 shows the plot of $\varepsilon_{\text {molar }}$ as a function of the wave number for as-deposited PTCDI thin films. Intensities of all the absorption bands are evaluated by measuring their oscillator strength, $f$, which is found to be proportional to the area under the absorption peak shapes. The oscillator strength and the electric dipole strength, $q^{2}$, can be calculated by the following mathematical expressions [43]:

$$
\begin{gathered}
f=4.3 \times 10^{-9} \int \varepsilon_{\text {molar }}(v) d v, \\
q^{2}=\frac{1}{2500} \varepsilon_{\text {molar }}(v)\left(\frac{\Delta \lambda}{\lambda}\right),
\end{gathered}
$$

where $\Delta \lambda$ is the absorption half-band width. The calculated values of the oscillator strength and the electric dipole strength for as-deposited films are listed in Table 4.

The complex dielectric constant $\left(\varepsilon^{*}=\varepsilon_{1}-i \varepsilon_{2}\right)$, the real dielectric constant $\varepsilon_{1}=\left(n^{2}-k^{2}\right)$, and the imaginary 
TABLE 4: Values of the center of the absorption band, oscillator strength, and electric dipole strength for as-deposited (PTCDI) thin films.

\begin{tabular}{lcc}
\hline Center of peaks $(\mathrm{eV})$ & $f$ & $q^{2}(A)^{2}$ \\
\hline 2.125 & 0.199 & 149.06 \\
2.544 & 1.24 & 83.5 \\
3.596 & 0.349 & 12.31 \\
5.799 & 4.3 & 103.37 \\
\hline
\end{tabular}

dielectric constant $\left(\varepsilon_{2}=2 n k\right)$, for PTCDI films, are illustrated in Figure 11. The real part generally relates to dispersion, while the imaginary part provides a measure to the dissipative rate of the light waves in the medium [44]. As observed, the real and imaginary parts show maximum related to the absorption values. It is also possible to calculate the volume and surface energy loss functions (VELF and SELF) by using the relations [40]

$$
\begin{gathered}
\operatorname{VELF}=\left(\frac{\varepsilon_{2}^{2}}{\varepsilon_{1}^{2}+\varepsilon_{2}^{2}}\right), \\
\operatorname{SELF}=\frac{\varepsilon_{2}^{2}}{\left(\left(\varepsilon_{1}+1\right)^{2}+\varepsilon_{2}^{2}\right)} .
\end{gathered}
$$

The results are shown in Figure 12.

\section{Conclusions}

The nanocrystalline PTCDI films were prepared by conventional thermal evaporation. The optical constants (refractive index $n$; extinction coefficient $k$; dielectric constant $\varepsilon$ ) were calculated. The refractive index spectra of the films showed both normal and anomalous dispersions. The dissipation factor exhibits various relaxation peaks due to the nanosized structure of the films which decreases the vibrational internal interval. The type of the electronic transition responsible for optical properties is indirect allowed transition. The refractive index dispersion parameters were obtained in the nonabsorbing region using the single-oscillator model. This change between the obtained lattice and high-frequency dielectric constant may be attributed to the free carrier contribution to the dispersion. All the results are self-consistent in the paper. This work would benefit the fabrication and investigation of organic solar cell and photovoltaic devices.

\section{References}

[1] J. Z. Sun, "High charge mobility of a perylene bisimide dye with hydrogen-bond formation group," Chinese Chemical Letters, vol. 16, no. 9, pp. 1201-1204, 2005.

[2] C. W. Struijk, A. B. Sieval, J. E. J. Dakhorst et al., "Liquid crystalline perylene diimides: architecture and charge carrier mobilities," Journal of the American Chemical Society, vol. 122, no. 45, pp. 11057-11066, 2000.

[3] P. R. L. Malenfant, C. D. Dimitrakopoulos, J. D. Gelorme et al., "N-type organic thin-film transistor with high field- effect mobility based on a $\mathrm{N}, \mathrm{N}^{\prime}$-dialkyl-3,4,9,10-perylene tetracarboxylic diimide derivative," Applied Physics Letters, vol. 80, no. 14, pp. 2517-2519, 2002.

[4] M. M. Shi, H. Z. Chen, and M. Wang, "Influences of different fluorinated substituents on electron mobility of perylene diimides," Acta Chimica Sinica, vol. 64, no. 8, pp. 721-726, 2006.

[5] M. M. Ling, Z. Bao, P. Erk, M. Koenemann, and M. Gomez, "Complementary inverter using high mobility airstable perylene di-imide derivatives," Applied Physics Letters, vol. 90, no. 9, Article ID 093508, 3 pages, 2007.

[6] S. H. Kim, Y. S. Yang, J. H. Lee et al., "Organic field-effect transistors using perylene," Optical Materials, vol. 21, no. 13, pp. 439-443, 2003.

[7] Y. Kim, I. Chung, Y. C. Kim, and J. Yu, "Mobility of electrons and holes in a liquid crystalline perylene diimide thin film with time of flight technique," Chemical Physics Letters, vol. 398, no. 4-6, pp. 367-371, 2004.

[8] Z. An, J. Yu, S. C. Jones et al., "High electron mobility in roomtemperature discotic liquid-crystalline perylene diimides," Advanced Materials, vol. 17, no. 21, pp. 2580-2583, 2005.

[9] Z. Chen, M. G. Debije, T. Debaerdemaeker, P. Osswald, and F. Würthner, "Tetrachloro-substituted perylene bisimide dyes as promising $n$-type organic semiconductors: studies on structural, electrochemical and charge transport properties," ChemPhysChem, vol. 5, no. 1, pp. 137-140, 2004.

[10] R. J. Chesterfield, J. C. McKeen, C. R. Newman et al., "Organic thin film transistors based on $\mathrm{N}$-alkyl perylene diimides: charge transport kinetics as a function of gate voltage and temperature," Journal of Physical Chemistry B, vol. 108, no. 50, pp. 19281-19292, 2004.

[11] J. H. Schön, C. Kloc, and B. Batlogg, "Perylene: a promising organic field-effect transistor material," Applied Physics Letters, vol. 77, no. 23, pp. 3776-3778, 2000.

[12] H. Z. Chen, M. M. Ling, X. Mo, M. M. Shi, M. Wang, and Z. Bao, "Air stable $n$ organic semiconductors for thin film transistors based on fluorinated derivatives of perylene diimides," Chemistry of Materials, vol. 19, no. 4, pp. 816-824, 2007.

[13] A. J. Breeze, A. Salomon, D. S. Ginley, B. A. Gregg, H. Tillmann, and H. H. Hörhold, "Polymer-perylene diimide heterojunction solar cells," Applied Physics Letters, vol. 81, no. 16, pp. 3085-3087, 2002.

[14] L. Schmidt-Mende, A. Fechtenkötter, K. Müllen, E. Moons, R. H. Friend, and J. D. MacKenzie, "Self-organized discotic liquid crystals for high-efficiency organic photovoltaics," Science, vol. 293, no. 5532, pp. 1119-1122, 2001.

[15] A. Yakimov and S. R. Forrest, "High photovoltage multipleheterojunction organic solar cells incorporating interfacial metallic nanoclusters," Applied Physics Letters, vol. 80, no. 9, pp. 1667-1669, 2002.

[16] L. Tan, M. D. Curtis, and A. H. Francis, "Characterization of organic $\mathrm{p} / \mathrm{n}$ junction photodiodes based on poly(alkylthiophene)/perylene diimide bilayers," Chemistry of Materials, vol. 15, no. 11, pp. 2272-2279, 2003.

[17] P. Peumans, S. Uchida, and S. R. Forrest, "Efficient bulk heterojunction photovoltaic cells using small-molecular-weight organic thin films," Nature, vol. 425, no. 6954, pp. 158-162, 2003.

[18] H. Z. Chen, M. M. Shi, T. Aernouts, M. Wang, G. Borghs, and P. Heremans, "A novel organic n-type material: fluorinated perylene diimide," Solar Energy Materials and Solar Cells, vol. 87, no. 1-4, pp. 521-527, 2005. 
[19] Y. Shibano, T. Umeyama, Y. Matano, and H. Imahori, "Electron-donating perylene tetracarboxylic acids for dyesensitized solar cells," Organic Letters, vol. 9, no. 10, pp. 19711974, 2007.

[20] L. Yang, M. Shi, M. Wang, and H. Z. Chen, "Self-assembled helical nano structures from an asymmetrical perylene diimide," Chinese Chemical Letters, vol. 19, no. 10, pp. 12601263, 2008.

[21] R. S. Loewe, K. Y. Tomizaki, W. J. Youngblood, Z. Bo, and J. S. Lindsey, "Synthesis of perylene_-porphyrin building blocks and rod-like oligomers for light-harvesting applications," Journal of Materials Chemistry, vol. 12, no. 12, pp. 3438-3451, 2002.

[22] K. Y. Tomizaki, R. S. Loewe, C. Kirmaier et al., "Synthesis and photophysical properties of light-harvesting arrays comprised of a porphyrin bearing multiple perylene-monoimide accessory pigments," Journal of Organic Chemistry, vol. 67, no. 18, pp. 6519-6534, 2002.

[23] X. Li, L. E. Sinks, B. Rybtchinski, and M. R. Wasielewski, "Ultrafast aggregate-to-aggregate energy transfer within selfassembled light-harvesting columns of zinc phthalocyanine tetrakis(perylenediimide)," Journal of the American Chemical Society, vol. 126, no. 35, pp. 10810-10811, 2004.

[24] B. Rybtchinski, L. E. Sinks, and M. R. Wasielewski, "Combining light-harvesting and charge separation in a self-assembled artificial photosynthetic system based on perylenediimide chromophores," Journal of the American Chemical Society, vol. 126, no. 39, pp. 12268-12269, 2004.

[25] A. M. Ramos, E. H. A. Beckers, T. Offermans, S. C. J. Meskers, and R. A. J. Janssen, "Photoinduced multistep electron transfer in an okligoaniline-oligo(p-phenylene vinylene)-perylene diimide molecular array," Journal of Physical Chemistry A, vol. 108, no. 40, pp. 8201-8211, 2004.

[26] M. A. Angadi, D. Gosztola, and M. R. Wasielewski, "Organic light emitting diodes using poly(phenylenevinylene) doped with perylenediimide electron acceptors," Materials Science and Engineering B, vol. 63, no. 3, pp. 191-194, 1999.

[27] P. Ranke, I. Bleyl, J. Simmerer, D. Haarer, A. Bacher, and H. W. Schmidt, "Electroluminescence and electron transport in a perylene dye," Applied Physics Letters, vol. 71, no. 10, pp. 13321334, 1997.

[28] S. Alibert-Fouet, S. Dardel, H. Bock et al., "Electroluminescent diodes from complementary discotic benzoperylenes," ChemPhysChem, vol. 4, no. 9, pp. 983-985, 2003.

[29] P. Schouwink, A. H. Schäfer, C. Seidel, and H. Fuchs, "Influence of molecular aggregation on the device properties of organic light emitting diodes," Thin Solid Films, vol. 372, no. 1, pp. 163-168, 2000.

[30] T. Zukawa, S. Naka, H. Okada, and H. Onnagawa, "Organic heterojunction phototransistor," Journal of Applied Physics, vol. 91, no. 3, p. 1171, 2002.

[31] M. Tengelin-Nilsson, L. Ilver, and J. Kanski, "Photoelectron spectroscopic studies of thin PTCDA layers on TiSe 2 ," Organic Electronics, vol. 3, no. 2, pp. 73-79, 2002.

[32] P. M. Kazmaier and R. Hoffman, "A theoretical study of crystallochromy. Quantum interference effects in the spectra of perylene pigments," Journal of the American Chemical Society, vol. 116, no. 21, pp. 9684-9691, 1994.

[33] L. Gross, C. Seidel, and H. Fuchs, "Organic monolayers with uniform domain orientation and reduced antiphase boundaries-MBE of perylene on $\mathrm{Au}(110)$," Organic Electronics, vol. 3, no. 1, pp. 1-7, 2002.

[34] S. Tolansky, Multiple Beam Interferometry Surfaces and Films, Oxford University Press, Oxford, UK, 1988.
[35] O. Stenzel, S. Wilbrant, A. Stendal, U. Beckers, K. Voigtsberger, and C. von Bor-czskowski, "The incorporation of metal clusters into thin organic dye layers as a method for producing strongly absorbing composite layers: an oscillator model approach to resonant metal cluster absorption," Journal of Physics D, vol. 28, no. 10, article 2154, 1995.

[36] S. H. Wemple and M. DiDomenico, "Optical dispersion and the structure of solids," Physical Review Letters, vol. 23, no. 20, pp. 1156-1160, 1969.

[37] S. H. Wemple and M. DiDomenico, "Behavior of the electronic dielectric constant in covalent and ionic materials," Physical Review B, vol. 3, no. 4, pp. 1338-1351, 1971.

[38] N. K. Sahoo and K. V. S. R. Apparao, "Process-parameter optimization of $\mathrm{Sb}_{2} \mathrm{O}_{3}$ films in the ultraviolet and visible region for interferometric applications," Applied Physics A, vol. 63, no. 2, pp. 195-202, 1996.

[39] S. H. Wemple, "Refractive-index behavior of amorphous semiconductors and glasses," Physical Review B, vol. 7, no. 8, pp. 3767-3777, 1973.

[40] M. M. El-Nahass, Z. El-Gohary, and H. S. Soliman, "Structural and optical studies of thermally evaporated CoPc thin films," Optics and Laser Technology, vol. 35, no. 7, pp. 523-531, 2003.

[41] D. Cahen, A. Kahn, and E. Umbach, "Energetics of molecular interfaces," Materials Today, vol. 8, no. 7, pp. 32-41, 2005.

[42] M. Dhanam, R. Balasundaraprabhu, S. Jayakumar, P. Gopalakrishnan, and M. D. Kannan, "Preparation and study of structural and optical properties of chemical bath deposited copper indium diselenide thin films," Physica Status Solidi A, vol. 191, no. 1, pp. 149-160, 2002.

[43] M. M. El-Nahass, A. A. Atta, H. E. A. El-Sayed, and E. F. M. El-Zaidia, "Structural and optical properties of thermal evaporated magnesium phthalocyanine $(\mathrm{MgPc})$ thin films," Applied Surface Science, vol. 254, no. 8, pp. 2458-2465, 2008.

[44] K. R. Rajesh and C. S. Menon, "Estimation of the refractive index and dielectric constants of magnesium phthalocyanine thin films from its optical studies," Materials Letters, vol. 53, no. 4, pp. 329-332, 2002. 

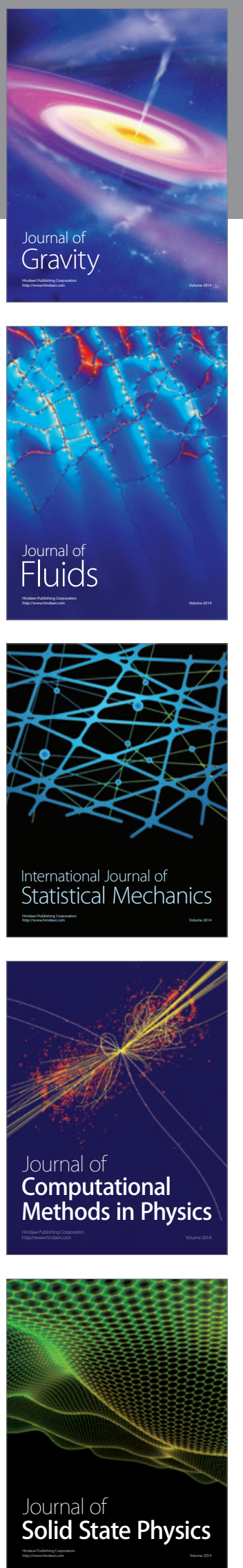

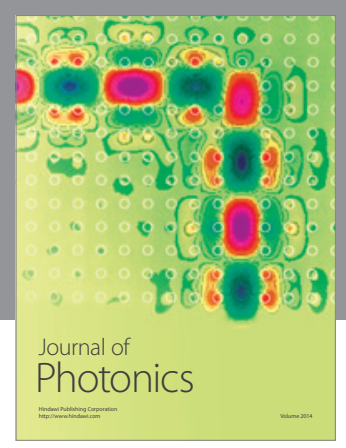

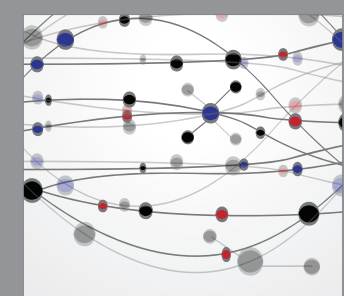

The Scientific World Journal
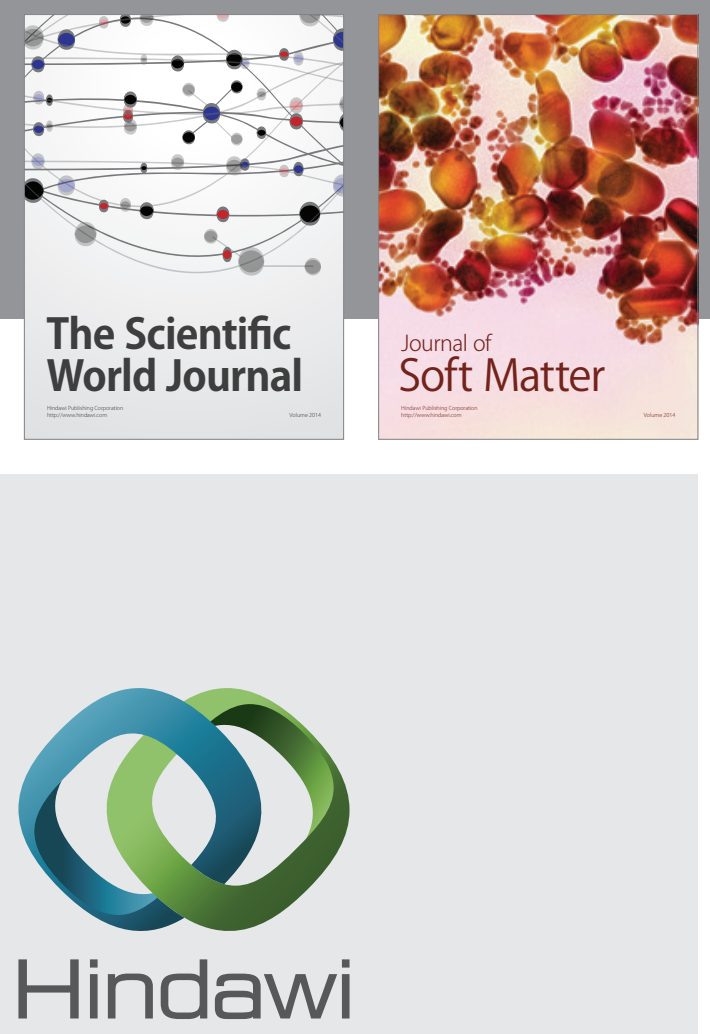

Submit your manuscripts at

http://www.hindawi.com
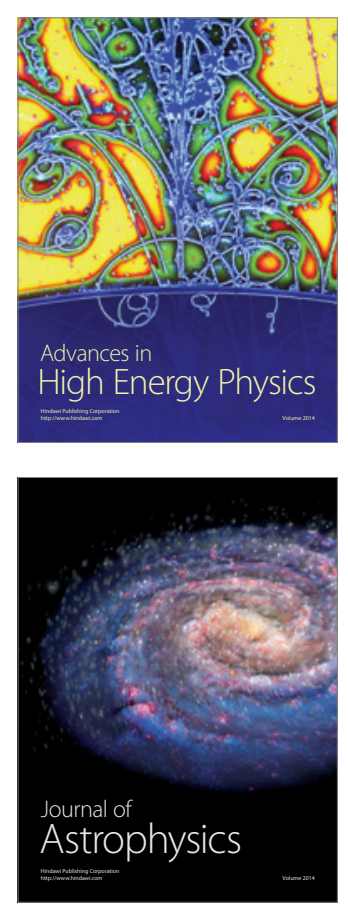
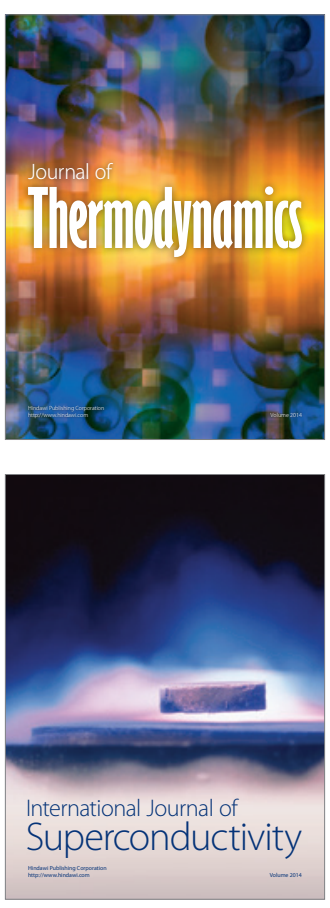
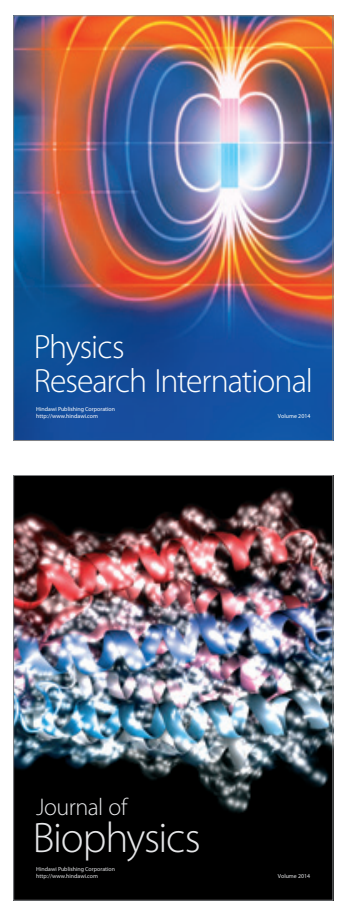
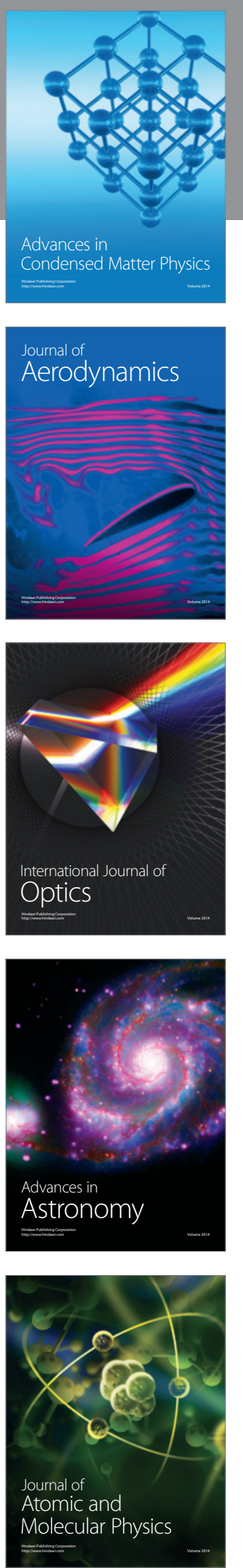